

\title{
Effect of carbapenem resistance on outcomes of bloodstream infection caused by Enterobacteriaceae in low-income and middle-income countries (PANORAMA): a multinational prospective cohort study
}

\author{
Andrew J Stewardson MBBS ${ }^{\text {abc }}{ }^{\dagger}$ Kali svarMarimuthu MBBS adeft Sharmila Sengupta MDg \\ Arthur Allignol PhDi Maisra El-Bouseary PhDjk Maria J Carvalho PhDk Brekhna Hassan PhDk \\ Monica ADelgado-Ramirez MD Anita Arora MD ${ }^{m}$ Ruchika Bagga MD ${ }^{n}$ Alex K Owusu-Ofori MD ${ }^{\circ}$ \\ Joseph O Ovosi MBBS ${ }^{p}$ Shamsudin Aliyu MBBS ${ }^{q}$ Hala Saad MDr Prof Souha S Kanj MDr \\ Prof Basudha Khanal MD ${ }^{s}$ Prof Balkrishna Bhattarai MD ${ }^{t}$ Samir K Saha PhD ${ }^{\mathrm{u}}$ Jamal Uddin MPH ${ }^{\mathrm{v}}$ \\ Purabi Barman MDw Latika Sharma MD Tarek El-Banna PhD ${ }^{\mathrm{j}}$ Rabaab Zahra PhDy \\ Mansab Ali Saleemi MPhily Amarjeet Kaur MD ${ }^{\text {h }}$ Kenneth Iregbu FWACPz
} Nkolika S C Uwaezuoke FWACP ${ }^{z}$ Pierre Abi Hanna MD ${ }^{\text {aa }}$ Rita Feghali MD ${ }^{\text {ab }}$ Prof Ana LCorrea MD ${ }^{\text {ac }}$ Maria IMunera MDad Thi Anh ThuLe MDa Thi Thanh Nga Tran MDaf Chimanjita Phukan MDag Chiranjita Phukan MD ${ }^{\text {ah }}$ Sandra L Valderrama-Beltrán MD ${ }^{\text {ai }}$ Prof Carlos Alvarez-Moreno MDaj Prof Timothy R Walsh DSck Prof Stephan Harbarth MDa

a Infection Control Program, University of Geneva Hospitals and Faculty of Medicine, WHO Collaborating Center, Geneva, Switzerland

b Department of Infectious Diseases, The Alfred Hospital and Central Clinical School, Monash University, Melbourne, VIC, Australia

c Harvard TH Chan School of Public Health, Harvard University, Boston, MA, USA

d Department of Infectious Diseases, Tan Tock Seng Hospital, Singapore

e National Centre for Infectious Diseases, Singapore

f Yong Loo Lin School of Medicine, National University of Singapore, Singapore

g Clinical Microbiology and Infection Control, Medanta, The Medicity Hospital, Delhi NCR, India

h Clinical Microbiology, Medanta, The Medicity Hospital, Delhi NCR, India

I Institute of Statistics, Ulm University, Ulm, Germany

j Pharmaceutical Microbiology, Tanta University, Tanta, Egypt

k Institute of Infection and Immunity, School of Medicine, Cardiff University, University Hospital of Wales, Cardiff, UK

I "Dr Manuel Gea Gonzalez" General Hospital, Mexico City, Mexico

$m$ Fortis Healthcare Limited, Gurgaon, India

n Microbiology, Fortis Memorial Research Institute, Gurgaon, India

- Clinical Microbiology, Komfo Anokye Teaching Hospital, Kwame Nkrumah University of Science and Technology, Kumasi, Ghana

p Internal Medicine, Clinical Pharmacology and Therapeutics, Kaduna State University, Barau Dikko Teaching Hospital, Kaduna, Nigeria

a Medical Microbiology, Ahmadu Bello University, Ahmadu Bello University Teaching Hospital, Zaria, Nigeria

$r$ Internal Medicine, Division of Infectious Diseases, American University of Beirut Medical Center, Beirut, Lebanon

s Microbiology and Infectious Diseases, Bishweshwar Prasad Koirala Institute of Health Sciences, Dharan, Nepal

t Anaesthesiology and Critical Care, Bishweshwar Prasad Koirala Institute of Health Sciences, Dharan, Nepal

u Department of Microbiology, Dhaka Shishu Hospital, Dhaka, Bangladesh

$\checkmark$ Department of Microbiology, Child Health Research Foundation, Dhaka, Bangladesh

w Clinical Microbiology and Hospital Infection Control, BLK Super Specialty Hospital, New Delhi, India

× Clinical Microbiology, BLK Super Specialty Hospital, New Delhi, India

y Department of Microbiology, Quaid-i-Azam University, Islamabad, Pakistan

z Department of Medical Microbiology and Parasitology, National Hospital, Abuja, Nigeria

aa Department of Medicine, Rafik Hariri University Hospital, Beirut, Lebanon

ab Department of Laboratory Medicine, Rafik Hariri University Hospital, Beirut, Lebanon

ac Infectious diseases, Hospital Pablo Tobón Uribe, Medellín, Colombia

ad Medical Microbiology, Hospital Pablo Tobón Uribe, Medellín, Colombia

ae Infection Control, Cho Ray Hospital, Ho Chi Minh City, Vietnam

af Microbiology, Cho Ray Hospital, Ho Chi Minh City, Vietnam

ag Department of Microbiology, Gauhati Medical College and Hospital, Guwahati, India

ah Department of Medicine, Gauhati Medical College and Hospital, Guwahati, India

ai Infectious Diseases, Internal Medicine, Hospital Universitario San Ignacio, Pontificia Universidad Javeriana, Bogotá, Colombia

aj Infectious Diseases, Internal Medicine Department, Universidad Nacional de Colombia, Bogotá, Colombia 


\section{SUMMARY}

\section{Background}

Low-income and middle-income countries (LMICS) are under-represented in reports on the burden of antimicrobial resistance. We aimed to quantify the clinical effect of carbapenem resistance on mortality and length of hospital stay among inpatients in LMICs with a bloodstream infection due to Enterobacteriaceae.

\section{Methods}

The PANORAMA study was a multinational prospective cohort study at tertiary hospitals in Bangladesh, Colombia, Egypt, Ghana, India, Lebanon, Nepal, Nigeria, Pakistan, and Vietnam, recruiting consecutively diagnosed patients with carbapenem-susceptible Enterobacteriaceae (CSE) and carbapenem-resistant Entero-bacteriaceae (CRE) bloodstream infections. We excluded patients who had previously been enrolled in the study and those not treated with curative intent at the time of bloodstream infection onset. There were no age restrictions. Central laboratories in India and the UK did confirmatory testing and molecular characterisation, including strain typing. We applied proportional subdistribution hazard models with inverse probability weighting to estimate the effect of carbapenem resistance on probability of discharge alive and in-hospital death, and multistate modelling for excess length of stay in hospital. All patients were included in the analysis.

\section{Findings}

Between Aug 1, 2014, and June 30, 2015, we recruited 297 patients from 16 sites in ten countries: 174 with CSE bloodstream infection and 123 with CRE bloodstream infection. Median age was 46 years (IQR 15-61). Crude mortality was 20\% (35 of 174 patients) for patients with CSE bloodstream infection and 35\% (43 of 123 patients) for patients with CRE bloodstream infection. Carbapenem resistance was associated with an increased length of hospital stay (3.7 days, $95 \% \mathrm{Cl} 0.3-6.9)$, increased probability of inhospital mortality (adjusted subdistribution hazard ratio $1 \cdot 75,95 \% \mathrm{Cl} 1 \cdot 04-2 \cdot 94$ ), and decreased probability of discharge alive (0.61, 0.45-0.83). Multilocus sequence typing showed various clades, with marginal overlap between strains in the CRE and CSE clades.

\section{Interpretation}

Carbapenem resistance is associated with increased length of hospital stay and mortality in patients with bloodstream infections in LMICs. These data will inform global estimates of the burden of antimicrobial resistance and reinforce the need for better strategies to prevent, diagnose, and treat CRE infections in LMICs. 


\section{INTRODUCTION}

Antimicrobial resistance (AMR) represents a substantial global health and economic threat, with resistance to so-called last-line antibiotics, such as carbapenems, of most concern. ${ }^{1,2}$ An accurate estimate of the burden of AMR is needed to inform decisions about allocation of resources towards strategies such as infection control measures, antimicrobial stewardship, and development of new antimicrobials. ${ }^{3,4}$ To date, only a few small studies have assessed the effect of carbapenem resistance on health outcomes, with most studies done in high-income settings. ${ }^{5,6,7,8,9,10}$ Yet, it is likely that the burden is greatest in low-income and middle-income countries (LMICs). ${ }^{11} \mathrm{~A} 2011$ meta-analysis of health-careassociated infections in LMICs reported an almost complete absence of data regarding the effect of AMR, ${ }^{12}$ and $\mathrm{WHO}$ has identified a knowledge gap on the burden of AMR in this setting. ${ }^{2}$

We aimed to describe the clinical features, outcomes, and molecular epidemiology of bloodstream infections caused by carbapenem-resistant Enterobacteriaceae (CRE) compared with carbapenemsusceptible Enterobacteriaceae (CSE) in LMICs. Our primary objective was to quantify the effect of carbapenem resistance on in-hospital mortality and length of hospital stay among inpatients diagnosed with a bloodstream infection due to a member of the Enterobacteriaceae family in LMICs.

\section{METHODS}

\section{Study design and participants}

We did a multinational prospective cohort study, the PANORAMA study. We established the PANORAMA network of local investigators from hospitals that met the following criteria: location in a country with a low-income or middle-income economy (at the time of patient recruitment), ${ }^{13}$ tertiary-level hospital according to $\mathrm{WHO}$ definition, ${ }^{14}$ and microbiology laboratory able to detect CRE. ${ }^{15}$ Patients were eligible if diagnosed with a bloodstream infection caused by a member of the Enterobacteriaceae family. We excluded patients who had previously been enrolled in the study and those not treated with curative intent at the time of bloodstream infection onset. Patients were recruited from hospitals linked to the local investigators. The exposure of interest was carbapenem resistance. We used an apportionment ratio of $1: 1$ to improve study precision. ${ }^{16}$ Local investigators enrolled consecutive eligible patients during a 6 month study period up to an initial maximum of five patients with CSE bloodstream infections and five patients with CRE bloodstream infections (established by the local laboratory in each hospital). After recruiting the initial ten patients, centres were invited to include ten more patients (five CRE and five CSE) in an ongoing stepwise manner for a maximum of 6 months. Patients were followed-up until discharge or in-hospital death. Study data were collected and managed using REDCap electronic data capture tools hosted at the University of Melbourne, VIC, Australia. ${ }^{17}$ This study was approved by the human research ethics committee at each institution. In some cases, consent was obtained. More frequently, the need for consent was waived according to local human research ethics guidance.

\section{Outcomes}

The primary outcomes were in-hospital mortality and length of hospital stay. As in-hospital mortality and discharge alive are competing outcomes, we modelled the effect of carbapenem resistance on both. ${ }^{18}$ 


\section{Procedures}

Between Aug 1, 2014, and June 30, 2015, the local investigators collected the following patient-level covariates: age, sex, referral source (admission from long-term care facility, nursing home, or transfer from another hospital), severity of underlying illness (measured using the Charlson comorbidity index ${ }^{19}$ ), Pitt bacteraemia score ${ }^{20}$ at bloodstream infection onset, and intensive care unit admission or surgical procedure before the bloodstream infection. Sanitation was categorised by household latrine facilities (ie, open defecation), and education used as a surrogate for socioeconomic status. We collected the following information about bloodstream infections: onset date (first positive culture), Enterobacteriaceae species, polymicrobial infection, source of infection (ie, primary, central-line associated, or secondary bloodstream infection), and epidemiological attribution (ie, hospital acquired, community acquired, or health-care associated). ${ }^{21}$ Antibiotic treatment was recorded. Agents used from day 0 (onset date) to day 2 of infection were defined as initial treatment. Persistent bloodstream infection was defined as a positive follow-up blood culture containing the same Enterobacteriaceae after more than $48 \mathrm{~h}$ of active antimicrobial treatment, or in the case of a catheter-associated bloodstream infection, a positive blood-culture result more than 3 days after removing the catheter. Recurrent bloodstream infection was defined as bacteraemia due to the same microbiologically documented Enterobacteriaceae (ie, species and antimicrobial susceptibility pattern), developing after the discontinuation of treatment. Follow-up blood cultures were taken at the discretion of the treating clinicians.

All blood culture isolates were processed in the clinical microbiology laboratory of each participating hospital for identification and antibiotic susceptibility testing, using standard microbiology methods. Strains were screened for carbapenem non-susceptibility according to recommendations of the International Working Group. ${ }^{15}$ All laboratories participated in an established external quality assurance programme. When possible, bacterial strains were sent to one of two central study laboratories (Cardiff, UK, and New Delhi, India) for confirmatory testing and molecular characterisation.

Species identification of bacterial isolates was confirmed by matrix-assisted laser desorption/ionisation time-of-flight mass spectrometry (MALDI-TOF; Bruker Daltonik GmbH, Coventry, UK), according to manufacturer's instructions, and the acquired mass spectra were compared with mass spectra in the Bruker database using MALDI Biotyper 3.1 software. Antibiotic resistance profiles against different antibiotics were established by disc diffusion, and minimum inhibitory concentrations of ertapenem, meropenem, imipenem, and colistin were established by agar dilution. ${ }^{22}$ Tests and interpretation were done according to the European Committee on Antimicrobial Susceptibility Testing guidelines version 7.1 (2017), and Escherichia coli ATCC25922 was used as the control.

Bacterial isolates were screened for the presence of b/a $a_{\mathrm{NDM}}, b / a_{\mathrm{KPC}}, b / a_{\mathrm{oxa}-48-\mathrm{like}}$, and $b / a_{\mathrm{VIM}}$ by PCR, and for the presence of b/a $a_{\mathrm{MP}-1}$, bla $a_{\mathrm{MP}-2}$, and bla $a_{\mathrm{MP}-3}$ by multiplex and individual PCR; a subset was confirmed by sequencing. See the appendix for primer sequences, target genes, and PCR conditions.

Multilocus sequence typing (MLST) was done following published protocols for (1) E coli and (2) Klebsiella pneumoniae. eBURST analysis was done to assess relationships between the different isolates on the basis of their sequence types (ST), and associated epidemiological data using PHYLOViZ. 


\section{Statistical analysis}

The sample size estimate was based on an expected in-hospital mortality of $40 \%$ among exposed (CRE) patients and $20 \%$ among non-exposed (CSE) patients. ${ }^{6}$ Given an $\alpha$ value of 0.05 and power of $80 \%$, we required 91 patients in each group to test the hypothesis that mortality is higher among exposed patients than among non-exposed patients. We assumed an institution-level intra-cluster correlation of 0.01 and a sample size of ten from each institution; therefore, we needed an actual sample size of 100 patients in each group to reach an effective sample size of 91.

The effect of carbapenem resistance on the probability of mortality and discharge alive was estimated using proportional subdistribution hazards models (Fine and Gray models ${ }^{23}$ ), with time from infection as the timescale (time 0 is infection, with infection therefore included as a time-fixed covariate). Hospital stays were censored at 90 days. For both outcomes (mortality and discharge alive), two models were fitted. The base model adjusted only for time from admission to infection. We applied inverse probability weighting to this model to account for confounding. ${ }^{24}$ We computed a propensity score for each patient, representing their probability of being infected with a carbapenem-resistant strain, using boosted regression trees that included time from admission to infection, age, sex, Charlson comorbidity index, location before admission, Pitt bacteraemia score, bacterial organism, education, and sanitation as predictors. ${ }^{25}$ We then used Cox proportional subdistribution hazards models weighted by 1 divided by the propensity score for patients with CRE blood-stream infection and 1 divided by ( 1 minus propensity score) for patients with CSE bloodstream infection. Time from admission to infection was included as an independent variable for doubly robust estimation. To account for missing values, we imputed 50 datasets using multiple imputation by chained equations (mice package), did the analysis described above with each dataset, and summarised results using Rubin's rule (appendix). ${ }^{26,} 27,28$

We used multistate modelling to estimate excess length of hospital stay associated with carbapenem resistance. ${ }^{29}$ We considered time from admission as the timescale. All patients entered the model at the time of infection in one of two states: CSE bloodstream infection or CRE bloodstream infection. Left truncation was taken into account by the estimators. ${ }^{30}$ Length of hospital stay is determined by moving into a final (ie, absorbing) state representing the day of discharge or in-hospital death. The excess length of hospital stay was based on non-parametric estimators of the transition probabilities associated with the multistate model (appendix). ${ }^{30} \mathrm{SEs}$ and $\mathrm{Cls}$ were derived by bootstrap re-sampling runs.

We did several sensitivity analyses. First, to evaluate the effect of timescale and to supplement the Fine and Gray models, we used Cox proportional hazards models to estimate the effect of carbapenem resistance on instantaneous risk of mortality, discharge alive, and a combined endpoint (all-cause end-ofstay) using two timescales: time from admission and time from infection. Second, to address the possibility of misclassification bias, in addition to the primary analysis including all recruited patients (ie, complete cohort) all analyses described were repeated on the subset of patients whose bloodstream infection strain was transported to one of two central laboratories for confirmatory testing (ie, confirmed cohort). The analysis of the complete cohort, which could be considered an intention-to-treat analysis, is presented in the main text of this paper, whereas the results for the confirmed cohort and other statistical sensitivity analyses are presented in the appendix. Statistical analyses were done using $R$, version 3.3.2, including the survival, etm, mvna, twang, and mice packages.

\section{RESULTS}

16 hospitals agreed to participate in the study (appendix), comprising ten public and six private facilities, with a median of 400 acute care beds (IQR 270-1100). Hospitals were located in two countries with low- 
income economies (Bangladesh and Nepal), six with lower-middle-income economies (Egypt, Ghana, India, Nigeria, Pakistan, and Vietnam), and two with upper-middle-income economies (Colombia and Lebanon). 14 (88\%) of 16 hospitals had specialist units for neonates, 12 (75\%) for obstetrics, ten (63\%) for burns, nine (56\%) for solid-organ transplantation, and six (38\%) for bone-marrow transplantation.

We recruited 297 patients between Aug 1, 2014, and June 30, 2015: 174 with CSE bloodstream infection and 123 with CRE bloodstream infection (figure 1). 297 patients were included in the complete cohort (ie, primary analysis). There were no dropouts. Missing data are described for each variable in table 1. Median age of all patients was 46 years (IQR 15-61); 182 (61\%) participants were male and 115 (39\%) were female (table 1). Open defecation at home was reported by $20(8 \%)$ of 256 patients. Median ageadjusted Charlson comorbidity index was 2.0 (IQR 0.0-5.0), and median Pitt bacteraemia score was $2 \cdot 0$ (IQR 0.5-4.0)

In-hospital mortality occurred in 35 (20\%) of 174 patients with CSE bloodstream infection and in 43 (35\%) of 123 patients with CRE bloodstream infection. Carbapenem resistance was associated with increased probability of in-hospital mortality and decreased probability of discharge alive in both the base and inverse probability weighted models (table 2). From the multistate model, carbapenem resistance was associated with 3.7 days (95\% $\mathrm{Cl} 0.3-6.9)$ excess length of stay in hospital. The results of the confirmed cohort and sensitivity analyses are consistent with those of the primary analysis in the complete cohort (appendix). From the isolates sent for confirmatory testing, positive predictive values for local laboratory designation of carbapenem resistance were $87 \%$ (84 of 97) and negative predictive values for local laboratory designation of carbapenem resistance were $96 \%$ (106 of 110).

Exposure to health care before bloodstream infection onset was more frequent among patients with CRE bloodstream infection than those with CSE bloodstream infection. The median length of stay in hospital before bloodstream infection onset was 1 day (IQR 0-5) for patients with CSE bloodstream infection and 4 days (0-14) for patients with CRE bloodstream infection (figure 2). Furthermore, more patients with CRE bloodstream infection had been exposed to antibiotics, intensive care, and surgery within the 30 days before bloodstream infection onset than had the patients with CSE bloodstream infection (table 1)

Antibiotic treatment is summarised in the appendix. Among 110 patients with CRE bloodstream infection, 36 (33\%) were prescribed a polymyxin as part of initial treatment. The antibiotic classes most frequently prescribed to patients with CRE bloodstream infection from day 3 onwards were carbapenems (55 [59\%] of 93) and polymyxins (48 [52\%] of 93), with 35 (38\%) of 93 patients in this group receiving an antibiotic from both of these classes. Carbapenems were the most commonly used antibiotic class for CSE bloodstream infection, with 65 (39\%) of 165 patients prescribed an agent from this class during the first 3 days of infection (ie, initial treatment period) and 63 (47\%) of 135 patients prescribed an agent from this class on subsequent days.

Although the incidence of septic shock among patients with CSE bloodstream infection (46 [26\%] of 174 patients) and CRE bloodstream infection (33 [27\%] of 123 patients) was similar, persistent bacteraemia was more frequently detected among patients with CRE bloodstream infection (21 [17\%] of 123 patients) than CSE bloodstream infection (14 [8\%] of 174 patients). Recurrent bacteraemia was also more frequently detected among patients with CRE bloodstream infection (eight [7\%] of 123 patients) than CSE bloodstream infection (five [3\%] of 174 patients).

Antibiotic susceptibility against 14 distinct antibiotics was established for $101 \mathrm{~K}$ pneumoniae isolates, $65 \mathrm{E}$ coli isolates, and 25 other Enterobacteriacae isolates (appendix). The antibiotic to which carbapenem- 
resistant isolates were most frequently susceptible was colistin: 36 (83\%) of $41 \mathrm{~K}$ pneumoniae isolates, all $15 \mathrm{E}$ coli isolates, and six (75\%) of eight isolates of other Enterobacteriaceae. All CRE isolates were nonsusceptible to cephalosporins and aztreonam. Resistance to non-carbapenem antibiotics was also widespread among CSE, particularly K pneumoniae. All carbapenem-susceptible $K$ pneumoniae, E coli, and other Enterobacteriaceae were susceptible to colistin, and amikacin (21 [57\%] of 37) and tigecycline (18 [53\%] of 34]) were active against more than half of the carbapenem-susceptible $K$ pneumoniae isolates.

In total, $109 \mathrm{~K}$ pneumoniae and $66 \mathrm{E}$ coli isolates were subjected to MLST. We identified $48 \mathrm{~K}$ pneumoniae STs. The most common were ST14 $(n=15)$, a non-virulent clone associated with capsular serotype K2, and ST231 ( $n=14$ ). 14 isolates belonged to clonal complex 258, including ST258, ST11, ST340, and ST2516. 35 STs were uniquely represented by one isolate. Additionally, five new allelic profiles (figure 3) were identified and have been added to the $K$ pneumoniae MLST database at Institut Pasteur (Paris, France). Among the $E$ coli isolates, 34 STs were identified. The most common was ST131 ( $n=n i n e)$, with 20 STs represented by one isolate. eBURST diagrams based on the STs obtained for K pneumoniae and E coli isolates are shown by carbapenem susceptibility in figure 3 and by geographical region in the appendix.

Among the 208 available isolates, b/a NDM $_{\text {was }}$ wast commonly identified carbapenemase-encoding gene; it was present in 35 carbapenem-resistant $K$ pneumoniae isolates ( 21 from south Asia, ten from Africa, and four from west Asia), ten carbapenem-resistant $E$ coli isolates (seven from south Asia, two from Africa, and one from west Asia), two other carbapenem-resistant Enterobacteriaceae, and one carbapenem-susceptible Klebsiella oxytoca (from Pakistan). bla oxA-48-like genes were identified in 34 carbapenem-resistant $K$ pneumoniae isolates ( 29 from south Asia, four from Africa, and one from west Asia) and one carbapenem-susceptible $K$ pneumoniae isolate (Africa). 11 carbapenem-resistant $E$ coli isolates carried blaOXA-48-like genes (nine from south Asia and two from west Asia). blakPc was detected in eight carbapenem-resistant $K$ pneumoniae isolates (seven from South America and one from south Asia) and one carbapenem-susceptible K pneumoniae isolate from South America. bla kPC was also found in six carbapenem-resistant $E$ coli isolates from south Asia and five carbapenem-resistant Enterobacter cloacae isolates. blavim genes were detected in three carbapenem-resistant $K$ pneumoniae isolates from Egypt. No

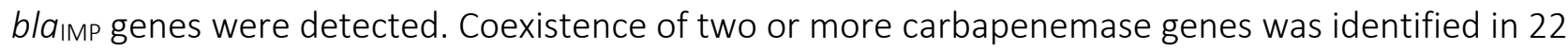
isolates. Carbapenemase-encoding genes were not found in one carbapenem-resistant $E$ coli isolate (Ghana), 11 carbapenem-resistant $K$ pneumoniae isolates (six from Ghana, three from India, and two from Egypt), and one Koxytoca isolate from Ghana.

\section{DISCUSSION}

These data show that in LMICs, patients with bloodstream infection caused by CRE have increased risk of death and length of stay in hospital compared with patients with bloodstream infection caused by CSE. Although this finding is not unexpected, data for the burden of AMR in LMICs are scarce, despite longstanding recognition of this knowledge gap. ${ }^{31,32}$ Such information is essential to justify the allocation of the resources required to strengthen laboratory, infection control, and antimicrobial stewardship capacity, and other strategies to address AMR in LMICs. ${ }^{33,34}$

Estimates of the global clinical burden of AMR need to include data from LMICs rather than being extrapolated from high-income countries for three reasons. First, there is an inverse relationship between national income and prevalence of drug-resistant infections. ${ }^{35}$ Potential reasons for this association include high population density, poor sanitation, contaminated water, environmental reservoirs, suboptimal implementation of infection control and antimicrobial stewardship interventions, selfmedication with over-the-counter antibiotics, and greater use of antibiotics for food production. ${ }^{32,35}$ Second, the clinical impact of each infection might be greater in LMICs because of barriers to health-care 
access, diagnostic testing, and therapeutic options. ${ }^{36}$ Finally, the molecular basis of carbapenem resistance varies by geographical region, ${ }^{37}$ so regional molecular data are required to evaluate the potential use of newer antimicrobial agents with activity against specific carbapenemase types.

The findings of this study are consistent with, and extend, the existing literature regarding the health impact and microbiology of carbapenem resistance among Enterobacteriaceae causing bloodstream infection in LMICs. ${ }^{38,39,40,41}$ Villegas and colleagues ${ }^{41}$ recruited patients from 11 hospitals in seven Latin American countries and found that CRE bloodstream infection was associated with a four-times increase in the odds of in-hospital mortality compared with CSE bloodstream infection. ${ }^{42}$ In this study, in-hospital mortality occurred in 35 (20\%) of 174 patients with CSE bloodstream infection and in 43 (35\%) of 123 patients with CRE bloodstream infection. We caution against directly comparing this result with recent studies from high-income settings without considering other differences in patient cohorts, including time period, patient acuity, and antibiotic susceptibilities. The INCREMENT study, ${ }^{43}$ done in 26 tertiary hospitals in ten countries, reported 30-day all-cause mortality of 43\% (189 of 437) in predominantly southern European patients with bloodstream infection due to carbapenemase-producing Enterobacteriaceae. The higher mortality than in our study might relate to changes in the management of carbapenemase-producing Enterobacteriaceae over time; 404 (92\%) of 437 patients in the INCREMENT study had infections from 2004 to 2011, and mortality was significantly higher among those patients than among those with infections from 2012 to 2013 (adjusted hazard ratio 1.43, 95\% Cl 1.02-2.01). Although several other factors are likely to be relevant, this secular trend is supported by smaller studies in highincome settings. 6, 7, 44 Additionally, INCREMENT excluded patients who had received appropriate antibiotics before bloodstream infection onset and used a different outcome measure to that used in our study (30-day vs in-hospital mortality).

The most common carbapenemase genes detected in this study were bla NDM $_{\text {and }}$ bla oxA-48-like, consistent with the large number of isolates from south Asia, which is the recognised epicentre of bla NDM $_{\text {and }}$ a region noted for more recent spread of OXA-48-like producing Enterobacteriaceae. ${ }^{45}$ As previously described, we found bla KPC $_{\text {in }}$ isolates from South America, ${ }^{45}$ but also in those from south Asia. No carbapenemase-encoding genes were identified in $13 \mathrm{CRE}$, suggesting the presence of other carbapenem resistance mechanisms.

The most common K pneumoniae STs were ST14 and ST231. K pneumoniae ST14 is a non-virulent ST that has been associated with capsular serotype $K 2$ and b/a ${ }_{\mathrm{NDM}}{ }^{46,47}$ a trend we also noted: eight of $15 \mathrm{~K}$ pneumoniae ST14 isolates from south Asia in this study carried blanDM. Similarly, we found bla genes in 12 of $14 \mathrm{~K}$ pneumoniae ST231 isolates (all from south Asia), as previously described. ${ }^{48} \mathrm{~K}$ pneumoniae ST258 is considered a high-risk international clone that is associated with clonal expansion of clades producing $K$ pneumoniae carbapenemase. ${ }^{45}$ In this study, five $K$ pneumoniae ST258 isolates carrying bla $\mathrm{KPC}_{\mathrm{BC}}$ genes were responsible for bloodstream infection cases in South America. 14 of $109 \mathrm{~K}$ pneumoniae isolates belonged to the clonal complex 258 (including ST258, ST11, ST340, and ST2516), which is associated with multidrug resistance and hospital dissemination. ${ }^{49}$ The most common E coli ST was ST131, a high-risk international clone associated with bla ${ }_{\mathrm{CTX}-\mathrm{M}-15 .}{ }^{45}$

A strength of this study is its prospective design, which facilitated accurate data collection. We also sought to provide valid estimates by explicitly accounting for competing outcomes and time-varying exposures. We did sensitivity analyses to explore the potential effect of misclassification of carbapenem susceptibility. To address the possibility of misclassification bias, we did all analyses on a subset of patients whose bacterial strains were characterised using phenotypic and molecular methods in one of two central study laboratories (ie, confirmed cohort). The multicentre nature of the study supports 
external validity. Finally, the molecular characterisation of dominant clades and carbapenemase genes adds detailed genetic information that is often lacking in clinical studies.

We acknowledge that this study has limitations. First, by fixing an equal ratio of susceptible and resistant infections, we are unable to directly establish the incidence of our exposure. This concession was made to increase the efficiency of our study (ie, to produce more precise estimates for a given sample size). Second, we restricted this study to tertiary institutions in large metropolitan areas to minimise information bias, assure feasibility, and reduce heterogeneity, but this strategy limits the generalisability of our findings. In rural hospitals, the clinical effect might be even greater due to the absence of adequate diagnostic and therapeutic infrastructure. Third, there is a substantial degree of heterogeneity present in this study with regard to hospital resources, patients (neonates vs adults), bacterial pathogens (E coli and $K$ pneumoniae), and resistance mechanisms. We did not power this study to do subgroup analyses stratified by these parameters. Finally, by comparing patients with bloodstream infection due to CSE with those with bloodstream infection due to CRE, we have estimated the effect of carbapenem resistance among patients with bloodstream infection. We are unable to estimate the effect of Enterobacteriaceae bloodstream infection (susceptible or resistant) in the absence of an uninfected group.

This study contributes to an improved understanding of the scale of the emerging threat of carbapenem resistance in LMICs. We provide detailed clinical data and molecular characterisation of strains, including high-risk multidrug-resistant clones with few remaining therapeutic options. We have produced a robust estimate of the mortality and excess length of hospital stay attributable to carbapenem resistance. When combined with incidence data, this study will inform estimates of the global health and economic burden of carbapenem resistance. In the future, affordable surveillance mechanisms, interventions to prevent infection, and management strategies should be developed to reduce the burden of bloodstream infections caused by CRE in LMICs. 


\section{RESEARCH IN CONTEXT}

\section{Evidence before this study}

We were provided with the results of an unpublished systematic review done by Pezzani and colleagues commissioned by WHO on the clinical burden of multidrug-resistant bacteria, which included studies published before July 15, 2017. This included six studies reporting the effect of carbapenem resistance on mortality and length of hospital stay among patients with bloodstream infection caused by Enterobacteriaceae spp in low-income and middle-income countries (LMICS). We added one publication by searching the PubMed database for studies published from July 1, 2007, to Nov 6, 2017, assessing the clinical effect of carbapenem resistance in LMICs. We used the search terms "Carbapenems" AND "Drug Resistance, Bacterial" OR "carbapenem-resistant" OR "carbapenemase-producing" OR "carbapenemase" AND "Enterobacteriaceae" AND "Bacteremia" OR "bloodstream infection" AND "Developing countries" OR "low-income" OR "middle-income". Altogether, this approach yielded seven publications: three were case series without a comparator group, three were single-centre studies (two in Taiwan and one in Brazil) with 75 participants or fewer, and one was a multicentre cohort study in seven Latin American countries. Although most studies reported higher mortality and increased length of hospital stay among patients with carbapenemresistant bloodstream infections, existing studies were underpowered and not able to account adequately for confounding and time-dependent bias. No published data were identified from low-income countries or from Africa, the Middle East, or south Asia.

\section{Added value of this study}

We add to previous evidence by including patients from a broader range of LMICs, and by using an analytical approach to account for the fact that discharge alive is a competing risk for in-hospital mortality and differences in follow-up between patients. We found that carbapenem resistance is associated with increased mortality and length of hospital stay among patients with bloodstream infections due to Enterobacteriaceae in LMICs. To our knowledge, these data represent the most detailed description to date of clinical and microbiological features of bloodstream infections due to carbapenem-resistant versus carbapenem-susceptible Enterobacteriaceae in LMICs. We also show the feasibility of studies to generate estimates of antimicrobial resistance in LMICs.

\section{Implications of all the available evidence}

The prevalence of resistance to third-generation cephalosporins among Enterobacteriaceae is unacceptably high, and carbapenems are increasingly being used to treat bloodstream infections. Carbapenem resistance is resulting in excess deaths and health expenditure in LMICs. In the absence of coordinated surveillance, this burden might go unrecognised, and appropriate interventions will be delayed. Although there is a need to improve surveillance of antimicrobial resistance in LMICs, this must be accompanied by strategies to limit the effect of resistance on patients with infections. Future research should develop affordable mechanisms for surveillance, evaluate the clinical effectiveness of interventions for infection prevention and antimicrobial stewardship, and optimise the management of bloodstream infections caused by carbapenem-resistant Enterobacteriaceae in LMICs. 


\section{REFERENCES}

1 World Bank Drug-resistant infections: a threat to our economic future World Bank, Washington, DC (2017)

2 WHO Antimicrobial resistance: global report on surveillance World Health Organization, Geneva (2014)

3 ME de Kraker, AJ Stewardson, S Harbarth Will 10 million people die a year due to antimicrobial resistance by 2050? PLoS Med, 13 (2016), p. e1002184

4 WHO Global priority list of antibiotic-resistant bacteria to guide research, discovery, and development of new antibiotics World Health Organization, Geneva (2017)

5 A Borer, L Saidel-Odes, K Riesenberg, et al. Attributable mortality rate for carbapenem-resistant Klebsiella pneumoniae bacteremia Infect Control Hosp Epidemiol, 30 (2009), pp. 972-976

6 G Patel, S Huprikar, SH Factor, SG Jenkins, DP Calfee Outcomes of carbapenem-resistant Klebsiella pneumoniae infection and the impact of antimicrobial and adjunctive therapies Infect Control Hosp Epidemiol, 29 (2008), pp. 1099-1106

7 M Tumbarello, P Viale, C Viscoli, et al. Predictors of mortality in bloodstream infections caused by Klebsiella pneumoniae carbapenemase-producing $K$. pneumoniae: importance of combination therapy Clin Infect Dis, 55 (2012), pp. 943-950

8 JR Zahar, JF Timsit, M Garrouste-Orgeas, et al. Outcomes in severe sepsis and patients with septic shock: pathogen species and infection sites are not associated with mortality Crit Care Med, 39 (2011), pp. 1886-1895

9 ME Falagas, GS Tansarli, DE Karageorgopoulos, KZ Vardakas Deaths attributable to carbapenemresistant Enterobacteriaceae infections Emerg Infect Dis, 20 (2014), pp. 1170-1175

10 El Kritsotakis, F Kontopidou, E Astrinaki, M Roumbelaki, E loannidou, A Gikas Prevalence, incidence burden, and clinical impact of healthcare-associated infections and antimicrobial resistance: a national prevalent cohort study in acute care hospitals in Greece Infect Drug Resist, 10 (2017), pp. 317-328

11 R Laxminarayan, DL Heymann Challenges of drug resistance in the developing world BMJ, 344 (2012), p. e1567

12 B Allegranzi, S Bagheri Nejad, C Combescure, et al. Burden of endemic health-care-associated infection in developing countries: systematic review and meta-analysis Lancet, 377 (2011), pp. 228-241

13 The World Bank World Bank list of economies

14 T Adam, DB Evans, CJ Murray Econometric estimation of country-specific hospital costs Cost Eff Resour Alloc, 1 (2003), p. 3

15 G Levy Hara, I Gould, A Endimiani, et al. Detection, treatment, and prevention of carbapenemaseproducing Enterobacteriaceae: recommendations from an International Working Group J Chemother, 25 (2013), pp. 129-140

16 KJ Rothman, S Greenland, TL Lash 10. Precision and statistics in epidemiologic studies KJ Rothman, S Greenland, TL Lash (Eds.), Modern epidemiology, Lippincott Williams \& Wilkins, Philadelphia, PA (2008), pp. $168-182$

17 PA Harris, R Taylor, R Thielke, J Payne, N Gonzalez, JG Conde Research electronic data capture (REDCap) - a metadata-driven methodology and workflow process for providing translational research informatics support J Biomed Inform, 42 (2009), pp. 377-381 
18 G De Angelis, A Allignol, A Murthy, et al .Multistate modelling to estimate the excess length of stay associated with meticillin-resistant Staphylococcus aureus colonisation and infection in surgical patients J Hosp Infect, 78 (2011), pp. 86-91

19 ME Charlson, P Pompei, KL Ales, CR MacKenzie A new method of classifying prognostic comorbidity in longitudinal studies: development and validation J Chronic Dis, 40 (1987), pp. 373-383

20 DL Paterson, WC Ko, A Von Gottberg, et al. International prospective study of Klebsiella pneumoniae bacteremia: implications of extended-spectrum beta-lactamase production in nosocomial infections Ann Intern Med, 140 (2004), pp. 26-32

21 ND Friedman, KS Kaye, JE Stout, et al. Health care-associated bloodstream infections in adults: a reason to change the accepted definition of community-acquired infections Ann Intern Med, 137 (2002), pp. 791-797

22 A Turlej-Rogacka, BB Xavier, L Janssens, et al. Evaluation of colistin stability in agar and comparison of four methods for MIC testing of colistin Eur J Clin Microbiol Infect Dis, 37 (2018), pp. 345-353

23 JP Fine, RJ Gray A proportional hazards model for the subdistribution of a competing risk J Am Stat Assoc, 94 (1999), pp. 496-509

24 MA Mansournia, DG Altman Inverse probability weighting BMJ, 352 (2016), p. i189

25 DF McCaffrey, G Ridgeway, AR Morral Propensity score estimation with boosted regression for evaluating causal effects in observational studies Psychol Methods, 9 (2004), pp. 403-425

26 S van Buuren, $\mathrm{K}$ Groothuis-Oudshoorn mice: Multivariate Imputation by chained equations in R J Stat Softw, 45 (2011), pp. 1-67

27 JW Bartlett, JM Taylor Missing covariates in competing risks analysis Biostatistics, 17 (2016), pp. 751-763

28 DB Rubin Inference and missing data Biometrika, 63 (1976), pp. 581-592

29 AJ Stewardson, A Allignol, J Beyersmann, et al. The health and economic burden of bloodstream infections caused by antimicrobial-susceptible and non-susceptible Enterobacteriaceae and Staphylococcus aureus in European hospitals, 2010 and 2011: a multicentre retrospective cohort study Euro Surveill, 21 (2016)

30 A Allignol, M Schumacher, J Beyersmann Estimating summary functionals in multistate models with an application to hospital infection data Comput Stat, 26 (2011), pp. 181-197

31 IN Okeke, R Laxminarayan, ZA Bhutta, et al. Antimicrobial resistance in developing countries. Part I: recent trends and current status Lancet Infect Dis, 5 (2005), pp. 481-493

32 RC Founou, LL Founou, SY Essack Clinical and economic impact of antibiotic resistance in developing countries: a systematic review and meta-analysis PLoS One, 12 (2017), p. e0189621

33 N Singh, V Manchanda Control of multidrug-resistant Gram-negative bacteria in low- and middleincome countries-high impact interventions without much resources Clin Microbiol Infect, 23 (2017), pp. 216-218

34 N Safdar, S Sengupta, JS Musuuza, et al. Status of the prevention of multidrug-resistant organisms in international settings: a survey of the Society for Healthcare Epidemiology of America Research Network Infect Control Hosp Epidemiol, 38 (2017), pp. 53-60

35 G Alvarez-Uria, S Gandra, R Laxminarayan Poverty and prevalence of antimicrobial resistance in invasive isolates Int J Infect Dis, 52 (2016), pp. 59-61 
36 R Laxminarayan, P Matsoso, S Pant, et al. Access to effective antimicrobials: a worldwide challenge Lancet, 387 (2016), pp. 168-175

37 RA Bonomo, EM Burd, J Conly, et al. Carbapenemase-producing organisms: a global scourge Clin Infect Dis, 66 (2018), pp. 1290-1297

38 HJ Chang, PC Hsu, CC Yang, et al. Risk factors and outcomes of carbapenem-nonsusceptible Escherichia coli bacteremia: a matched case-control study J Microbiol Immunol Infect, 44 (2011), pp. 125-130

39 SW Liu, HJ Chang, JH Chia, AJ Kuo, TL Wu, MH Lee Outcomes and characteristics of ertapenemnonsusceptible Klebsiella pneumoniae bacteremia at a university hospital in Northern Taiwan: a matched case-control study J Microbiol Immunol Infect, 45 (2012), pp. 113-119

40 L Correa, MD Martino, I Siqueira, et al. A hospital-based matched case-control study to identify clinical outcome and risk factors associated with carbapenem-resistant Klebsiella pneumoniae infection BMC Infect Dis, 13 (2013), p. 80

41 MV Villegas, CJ Pallares, K Escandon-Vargas, et al. Characterization and clinical impact of bloodstream infection caused by carbapenemase-producing Enterobacteriaceae in seven Latin American countries PLoS One, 11 (2016), p. e0154092

42 C Lim, E Takahashi, M Hongsuwan, et al. Epidemiology and burden of multidrug-resistant bacterial infection in a developing country Elife, 5 (2016), p. e18082

43 B Gutierrez-Gutierrez, E Salamanca, M de Cueto, et al. Effect of appropriate combination therapy on mortality of patients with bloodstream infections due to carbapenemase-producing Enterobacteriaceae (INCREMENT): a retrospective cohort study Lancet Infect Dis, 17 (2017), pp. 726-734

44 PD Tamma, KE Goodman, AD Harris, et al. Comparing the outcomes of patients with carbapenemase-producing and non-carbapenemase-producing carbapenem-resistant Enterobacteriaceae bacteremia Clin Infect Dis, 64 (2017), pp. 257-264

45 D van Duin, Y Doi The global epidemiology of carbapenemase-producing Enterobacteriaceae Virulence, 8 (2017), pp. 460-469

46 CG Giske, I Froding, CM Hasan, et al. Diverse sequence types of Klebsiella pneumoniae contribute to the dissemination of $b / a_{\mathrm{NDM}-1}$ in India, Sweden, and the United Kingdom Antimicrob Agents Chemother, 56 (2012), pp. 2735-2738

47 S Brisse, $V$ Passet, $A B$ Haugaard, et al. wzi gene sequencing, a rapid method for determination of capsular type for Klebsiella strains J Clin Microbiol, 51 (2013), pp. 4073-4078

48 MHF Abdul Momin, A Liakopoulos, LM Phee, DW Wareham Emergence and nosocomial spread of carbapenem-resistant OXA-232-producing Klebsiella pneumoniae in Brunei Darussalam J Glob Antimicrob Resist, 9 (2017), pp. 96-99

49 KE Holt, H Wertheim, RN Zadoks, et al. Genomic analysis of diversity, population structure, virulence, and antimicrobial resistance in Klebsiella pneumoniae, an urgent threat to public health Proc Natl Acad Sci USA, 112 (2015), pp. e3574-e3581 


\section{Figure 1. Trial profile}

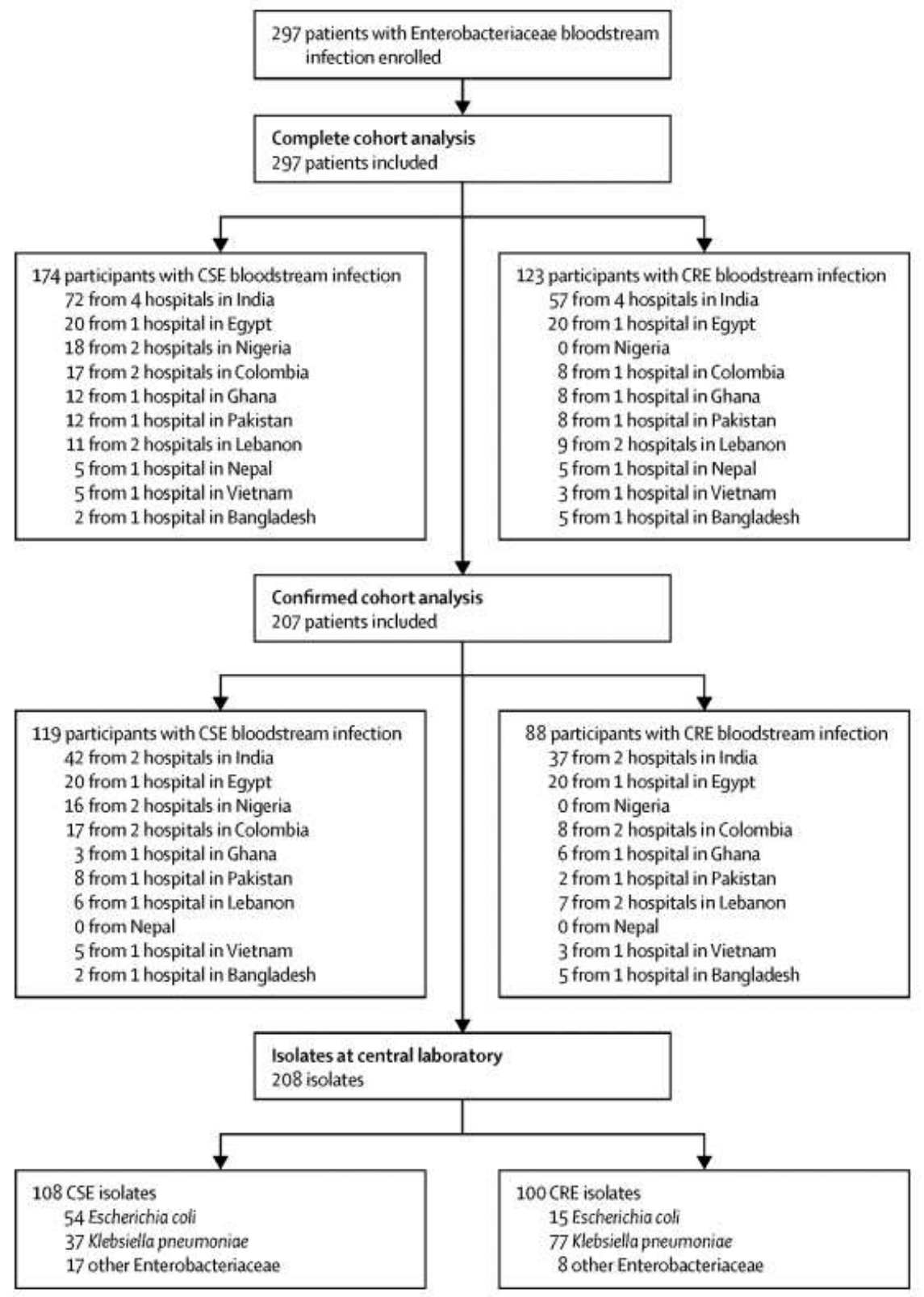

More than one isolate was available from some participants in the confirmed cohort. The complete cohort analysis included all patients. The confirmed cohort analysis included the subset of patients whose bloodstream infection strain was transported to a central study laboratory for confirmatory testing. CRE=carbapenem-resistant Enterobacteriaceae. CSE=carbapenemsusceptible Enterobacteriaceae. 
Figure 2. Time from hospital admission to bloodstream infection and outcome, stratified by carbapenem susceptibility

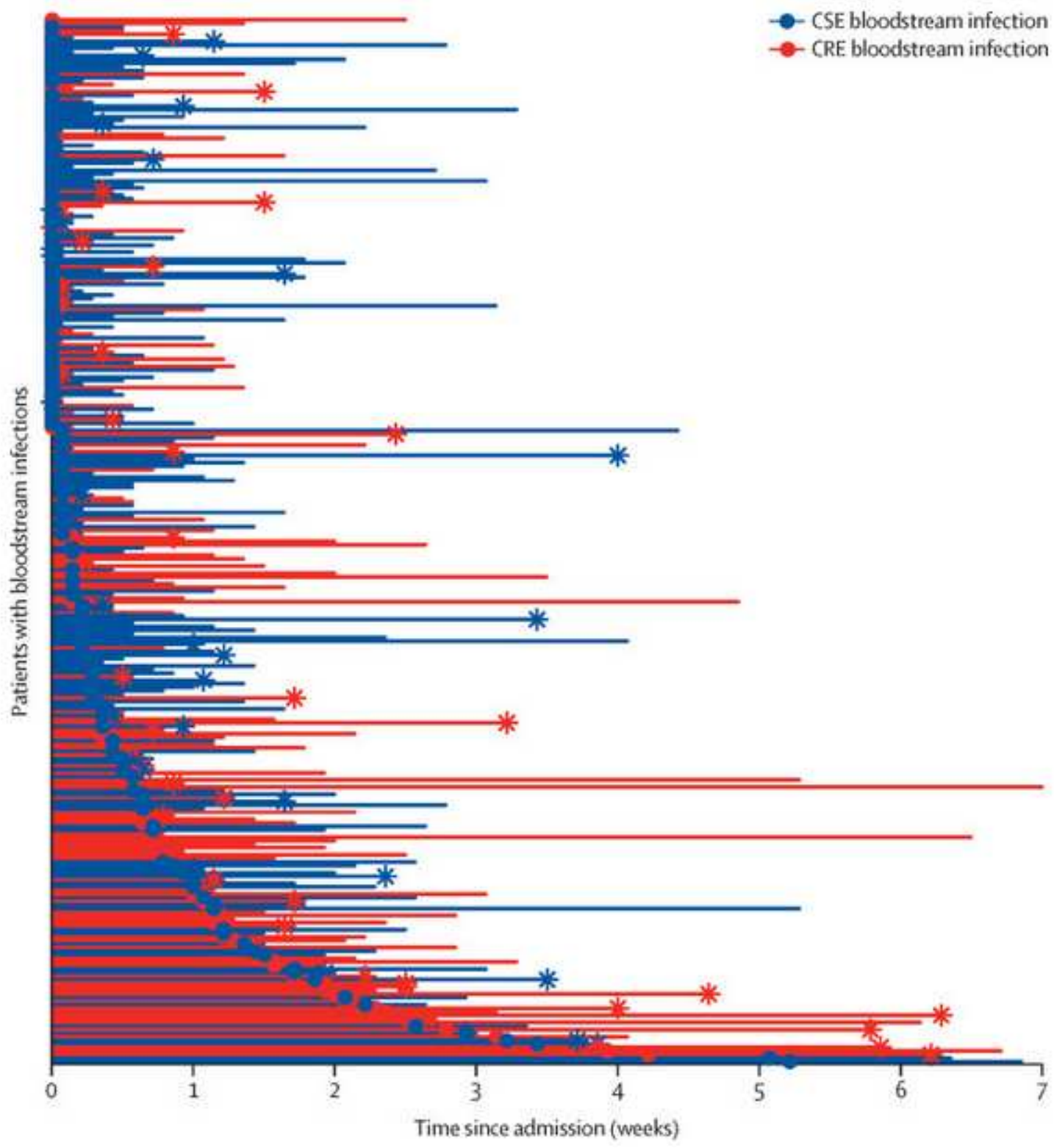

Each line represents one patient admission. Admissions are arranged vertically according to time from admission to bloodstream infection onset. Filled circles indicate bloodstream infection onset. Stars at the end of bars show mortality. CRE=carbapenem-resistant Enterobacteriaceae. CSE=carbapenemsusceptible Enterobacteriaceae. 
Figure 3. eBURST diagrams of Klebsiella pneumoniae (A) and Escherichia coli (B) isolates showing the relationship between isolates on the basis of their multilocus sequence typing and carbapenem susceptibility

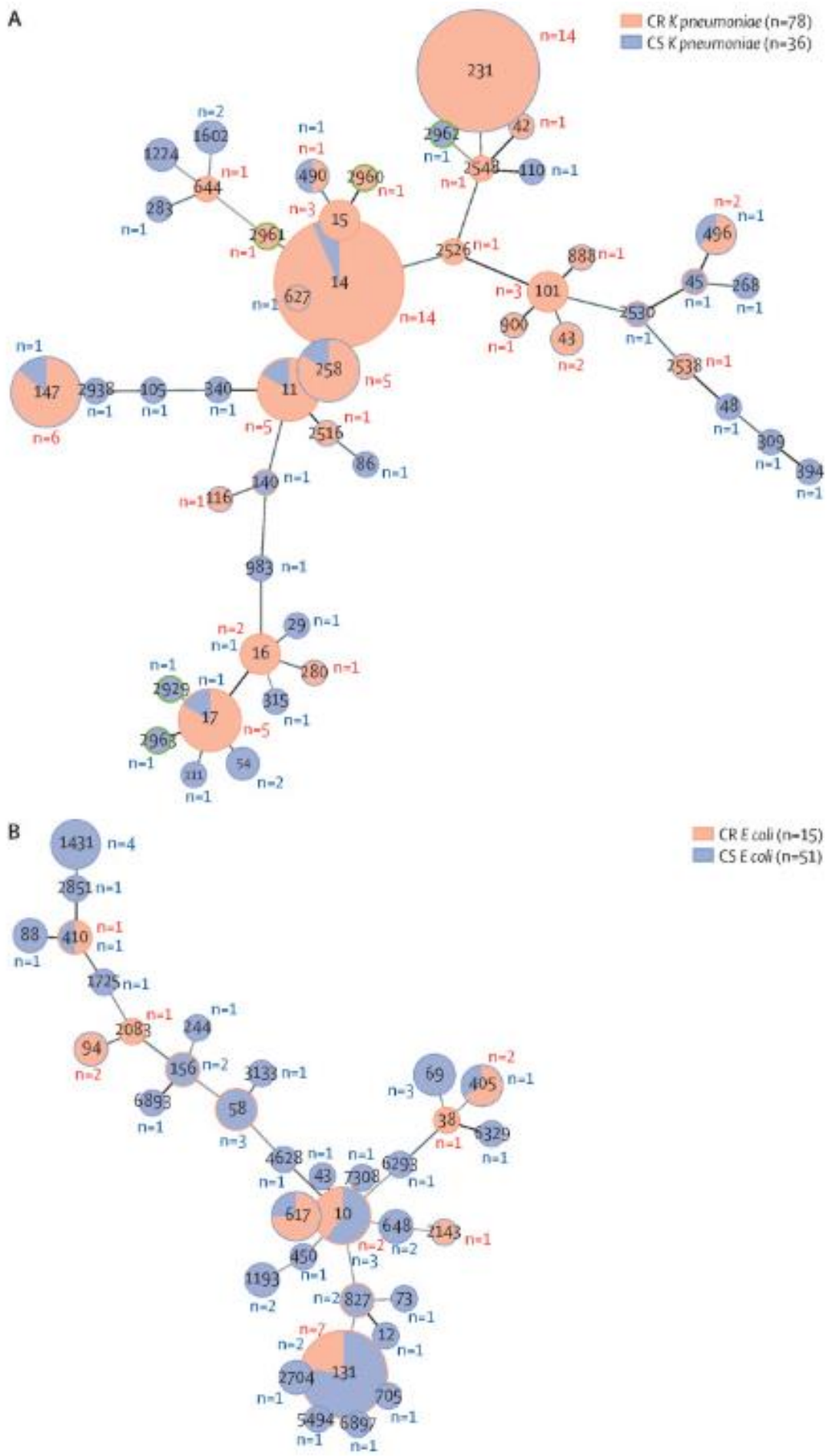

Each node within the tree represents a single ST. The size of the nodes is proportional to the number of isolates represented by said node. Within each node, the area of blue and orange represents the number of carbapenem-susceptible and carbapenem-resistant strains. Nodes are labelled with corresponding ST. Nodes representing new STs are delineated with a green circle. $\mathrm{ST}=$ sequence type. $\mathrm{CR}=$ carbapenem-resistant. $\mathrm{CS}=$ carbapenem-susceptible. 
Table 1. Patient characteristics

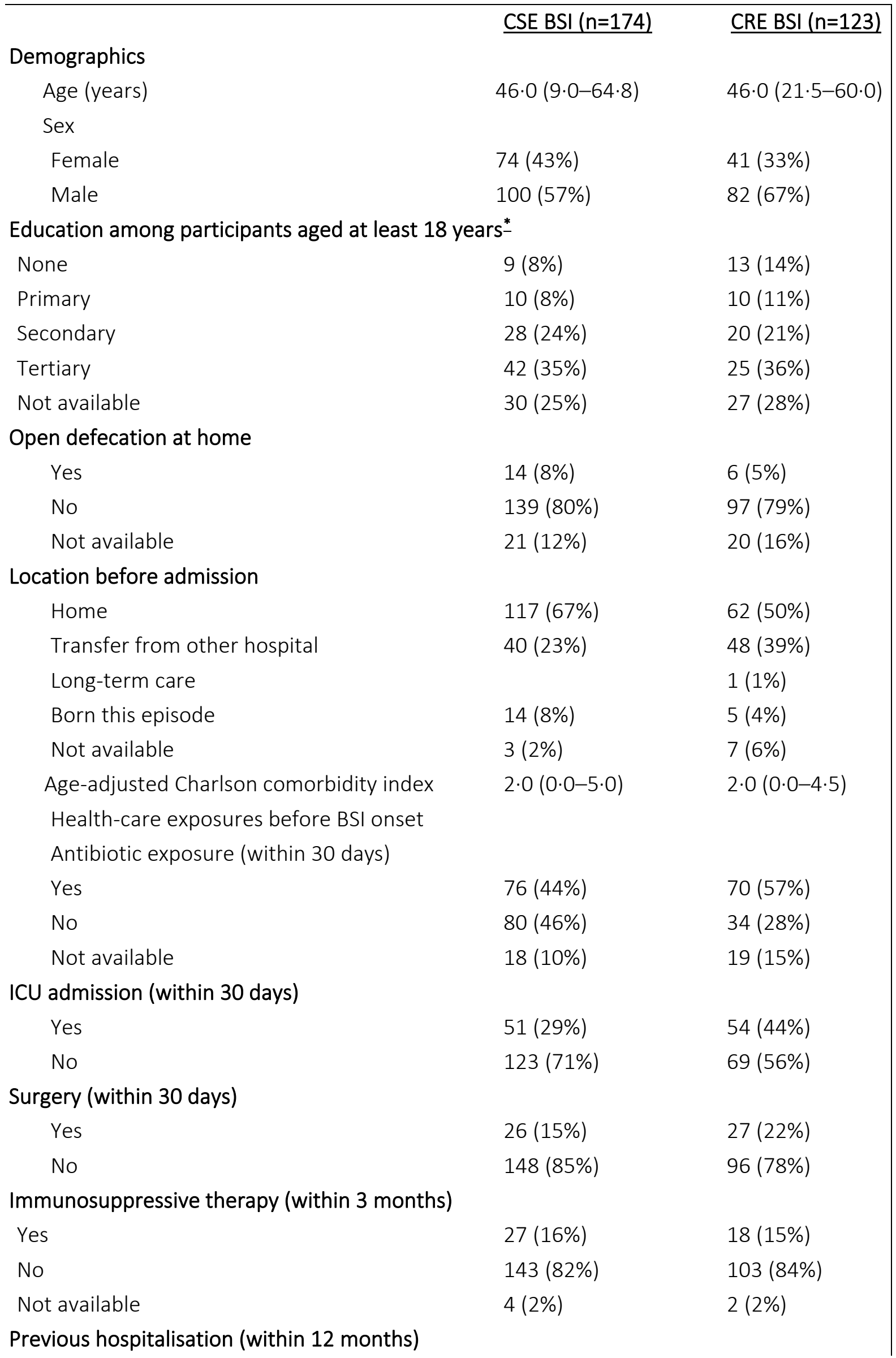




\begin{tabular}{|c|c|c|}
\hline & CSE BSI $(n=174)$ & CRE BSI $(n=123)$ \\
\hline Yes & $96(55 \%)$ & $77(63 \%)$ \\
\hline No & $77(44 \%)$ & $46(37 \%)$ \\
\hline Not available & $1(1 \%)$ & $0(0 \%)$ \\
\hline \multicolumn{3}{|l|}{ Qualifying Enterobacteriaceae } \\
\hline Escherichia coli & $83(48 \%)$ & $20(16 \%)$ \\
\hline Klebsiella species & $69(40 \%)$ & $85(69 \%)$ \\
\hline Other Enterobacteriaceae & $19(11 \%)$ & $10(8 \%)$ \\
\hline More than one Enterobacteriaceae & $3(2 \%)$ & $8(7 \%)$ \\
\hline ICU at time of BSI onset & $73(42 \%)$ & $68(55 \%)$ \\
\hline Pitt bacteraemia score at BSI onset ${ }^{\dagger}$ & $2 \cdot 0(0 \cdot 0-4 \cdot 0)$ & $2 \cdot 5(1 \cdot 0-5 \cdot 0)$ \\
\hline \multicolumn{3}{|l|}{ Epidemiological classification } \\
\hline Community acquired & $60(34 \%)$ & $11(9 \%)$ \\
\hline Hospital acquired & $69(40 \%)$ & $65(53 \%)$ \\
\hline Health-care associated with community onst & $25(14 \%)$ & $29(24 \%)$ \\
\hline Not available & $20(11 \%)$ & $18(15 \%)$ \\
\hline \multicolumn{3}{|l|}{ Source } \\
\hline Primary & $99(57 \%)$ & $59(48 \%)$ \\
\hline Central-line associated & $18(10 \%)$ & $26(21 \%)$ \\
\hline Secondary $\ddagger$ & $57(33 \%)$ & $38(31 \%)$ \\
\hline CNS & $1(2 \%)$ & $2(5 \%)$ \\
\hline Cardiovascular system infection & 0 & $1(3 \%)$ \\
\hline Gastrointestinal system infection & $14(25 \%)$ & $8(21 \%)$ \\
\hline Lower respiratory infection, other than pneums & $6(11 \%)$ & $3(8 \%)$ \\
\hline Pneumonia or ventilator-associated event & $12(21 \%)$ & $6(16 \%)$ \\
\hline Reproductive tract infection & $1(2 \%)$ & 0 \\
\hline Surgical site infection & $3(5 \%)$ & $4(11 \%)$ \\
\hline Skin and soft tissue infection & 0 & $9(24 \%)$ \\
\hline Urinary tract infection & $20(35 \%)$ & $5(13 \%)$ \\
\hline Total length of stay (days) & $11 \cdot 0(6 \cdot 0-24 \cdot 0)$ & $19 \cdot 0(10 \cdot 0-31 \cdot 5)$ \\
\hline
\end{tabular}

Data are $n(\%)$ or median (IQR). CSE=carbapenem-susceptible Enterobacteriaceae. BSI=bloodstream infection. CRE=carbapenem-resistant Enterobacteriaceae. ICU=intensive care unit.

- 119 patients with CSE BSI and 95 patients with CRE BSI were aged at least 18 years.

+ Not available for one patient with CSE BSI and one patient with CRE BSI.

$\ddagger$ For sources of secondary BSI, percentages represent the proportion of all secondary BSIs accounted for by that source (stratified by carbapenem susceptibility). 
Table 2. Effect of carbapenem resistance on in-hospital death and discharge alive from proportional subdistribution hazards models*

Subdistribution

$\underline{\mathrm{HR}(95 \% \mathrm{Cl})}$

In-hospital mortality

Base analysis

$1.68(1.07-2 \cdot 64)$

Inverse probability weighted analysis

$1.75(1.04-2 \cdot 94)$

Alive at discharge

Base analysis

$0.63(0.48-0.84)$

Inverse probability weighted analysis

$0.61(0.45-0.83)$

$\mathrm{HR}=$ hazard ratio

* Analysis done on the complete cohort; all models include time from admission to infection as a covariate 


\section{Role of the funding source}

The funder of the study had no role in study design, data collection, data analysis, data interpretation, or writing of the report. The corresponding author had full access to the data and had final responsibility for the decision to submit for publication.

\section{Declaration of interests}

AAl has been employed by Merck KGaA (Darmstadt, Germany) since August, 2017, and has shares in the company. CA-M reports grants, personal fees, and non-financial support from Merck Sharp \& Dohme, personal fees from Pfizer, and grants and personal fees from GlaxoSmithKline, outside the submitted work. SLV-B reports personal fees from Merck Sharp \& Dohme and Pfizer, outside the submitted work. SH reports grants from bioMérieux, during the conduct of the study, and personal fees from DNA Electronics and Sandoz, outside the submitted work. All other authors declare no competing interests.

\section{Acknowledgments}

The PANORAMA study was partly funded by an unrestricted research grant provided by bioMérieux (Marcy l'Etoile, France). AJS was supported by a National Health and Medical Research Council Early Career Fellowship (APP1141398). KM was supported by the Health Manpower Development Plan during the planning phase of this study. We gratefully acknowledge contributions from Benedetta Allegranzi (Patient Safety Program, WHO), Didier Pittet (Infection Control Program and WHO Collaborating Centre on Patient Safety, University of Geneva Hospitals), Nalini Singh (Division of Infectious Disease at Children's National Health System, Washington DC), Partha Pratim De (Head, Department of Laboratory Medicine, Tan Tock Seng Hospital), Fatima Modibbo (Department of Medical Microbiology, National Hospital Abuja), and Hemant Gupta (Qiagen). We are grateful to Evelina Tacconelli and Diletta Pezzani for providing the relevant results from their systematic review on the burden of AMR. 